\title{
Deadline Delay Constrained Multiuser Multicell Systems: Energy Efficient Scheduling
}

\author{
M. Majid Butt \\ Fraunhofer Heinrich Hertz Institute \\ Einsteinufer 37, 10587 Berlin, Germany \\ Email: majid.butt@hhi.fraunhofer.de
}

\begin{abstract}
This work considers the scheduling problem for the packets with strict maximum tolerable delay (deadline) constraint. We discuss a multiuser scheduling scheme which schedules the users based on their instantaneous channel conditions, packet deadline and backlog of the buffered packets. The scheme is analyzed in large system limit for the single and multicell cases. The aim is to minimize the system transmit energy while providing hard deadline guarantees for every packet. We analyze the effect of intercell interference on the deadline delay constrained systems and derive an upper bound on the achievable capacity for a given cell size. Numerical results provide the characterization of the operating region of a deadline delay constrained multicell system as a function of spectral efficiency.
\end{abstract}

\section{INTRODUCTION}

A large number of applications require minimum quality of service (QoS) guarantees in terms of maximum tolerable delay for the packet, called deadline. We study the problem with the objective of providing these guarantees at minimum system energy. Wireless sensor networks and green radio networks are the examples of such applications where minimization of energy consumption is extremely important.

In a multiuser environment, multiuser diversity is inherent in the system and many researchers exploit it opportunistically to solve various scheduling problems, e.g., the authors in [1] use multiuser diversity to provide statistical QoS in terms of data rate, delay bound, and delay bound violation probability. A similar work in [2] addresses the problem of scheduling multiple transmissions on the downlink with performance guarantees in terms of probabilities that the short term throughput exceeds a user specified throughput.

Energy-delay trade-off for the hard deadline delay constrained systems has been discussed in literature in different problem settings [3], [4]. The authors in [5] discuss the energy delay trade-off for a scheduler with full and partially shared information about the queue lengths of all the users.

A simple heuristic algorithm for the packet deadline constrained scheduling in a multiuser system has been introduced briefly in [6]. Backlog and channel dependent thresholds are computed numerically and an energy-delay tradeoff is demonstrated. In this work, we extend the results of [6] for a multicell system. As compared to Monte Carlo simulation based work in [6], this work analyzes the scheme for the multicell case in the large user limit and investigates the effect of intercell interference on the system energy specifically.
The rest of the paper is structured as follows. Section II introduces the system model. In Section III, we model and analyze the proposed scheduler in the large system limit for the single and multicell cases. We discuss optimization procedure in Section IV briefly. In Section V, we evaluate the numerical results for the scheduler in a multicell scenario and conclude with the main contributions of the work in Section VI.

\section{System Model}

We consider a multiple-access system with $K$ users per cell in a multicell system. The base stations are arranged on a uniform grid on a line. This simplified channel model is known as Wyner model [7]. The distance between two base stations is denoted by $D$.

Each user is provided a certain fraction of the total spectral efficiency available to the system. The required average rate $R$ for each user is $\frac{\Gamma}{K}$ where $\Gamma$ denotes the system spectral efficiency. We consider a multiband system with $M$ channels. $\Gamma$ is normalized by $M$ to get spectral efficiency per channel $C$. We consider an uplink scenario and assume perfect channel state information (CSI) on both transmitter and receiver sides. However, a user has no information of her channels to the base stations in neighboring cells.

Each user $k$ experiences a channel gain $g_{k}(l, j, t)$ at cell $j$ to cell $l$ in slot $t$. The channel gain is the product of path loss $s_{k}(l, j)$ and short-term fading $f_{k}(l, j, t)$, i.e., $g_{k}(l, j, t)=$ $s_{k}(l, j) f_{k}(l, j, t)$. We denote the short term fading $f_{k}(j, j, t)$ and the corresponding channel gain $g_{k}(j, j, t)$ in a user's own cell by $f_{k}(t)$ and $g_{k}(t)$, respectively. For a multi-band system of $M$ channels, short-term fading over the best channel is represented by, $f_{k}(t)=\max \left(f_{k}^{(1)}(t), f_{k}^{(2)}(t), \ldots, f_{k}^{(M)}(t)\right)$. The path loss depends on the distance between the transmitter and the receiver and remains constant within the time-scales considered in this work. In a multiple-cell system, path loss equals to $d_{k}(l, j)^{-\alpha}$ where distance $d_{k}(l, j), l \neq j$ is given by

$$
d_{k}(l, j)=|l-j| D \pm d_{k}(j, j)
$$

The sign for $d_{k}(j, j)$ depends on the location of the user in cell $j$ [7] and $\alpha$ denotes the path loss exponent.

Path loss and short-term fading are assumed to be independent. Short-term fading changes from slot to slot for every user and is independent and identically distributed across both the users and the slots; but remains constant within each time slot. This model is called block fading model. 
Let $E_{k}^{R}(t)$ and $E_{k}(t)$ denote the received and the transmitted energy for each user $k$ such that

$$
E_{k}^{R}(t)=g_{k}(t) E_{k}(t) .
$$

As multiple users can reach the deadline in the same time slot, we schedule multiple users simultaneously. The multiple scheduled users are separated by superposition coding. Let $\Delta_{m}$ be the set of users to be scheduled in frequency band $m$. Let $\psi_{k}^{(m)}$ be the permutation of the scheduled user indices for frequency band $m$ that sorts the channel gains in increasing order, i.e. $g_{\psi_{1}}^{(m)} \leq \cdots \leq g_{\psi_{k}}^{(m)} \leq \cdots \leq g_{\psi_{\left|\Delta_{m}\right|}}^{(m)}$. Then, the energy of user $\psi_{k}^{(m)}$ with rate $R_{\psi_{k}}^{(m)}$ is given by [7]

$$
E_{\psi_{k}}=\frac{N_{0}+I(l)}{g_{\psi_{k}}}\left[2^{\sum_{i \leq k} R_{\psi_{i}}}-2^{\sum_{i<k} R_{\psi_{i}}}\right] .
$$

where $N_{0}$ denotes the noise power spectral density and $I(l)$ is the outer cell interference on cell $l$ given by

$$
I(l)=\sum_{j \neq l} \sum_{k=1}^{K} g_{k}(l, j) E_{k}(j)
$$

It is modeled as Gaussian noise. Every user takes the scheduling decision independent of the other users but the required energy is assigned by a central scheduler which requires CSI of the other users. The energy assignment in (3) results in the minimum total transmit energy for the scheduled users [7].

\section{Modeling And ANALysis of the Scheduler}

The scheduling decision for a user $k$ in a time slot $t$ is based on the delay of the oldest packet in the buffer and instantaneous short-term fading $f_{k}(t)$. A group of users experiencing relatively large short-term fading is scheduled for transmission simultaneously. When a user is scheduled for transmission, she empties her buffer by scheduling all the packets buffered. If waiting time of the oldest buffered packet of a user is equal to the hard deadline and the fading is not good enough to allow transmission of the full buffer, the packet reaching the deadline is scheduled and a channel is assigned regardless of her instantaneous fading state.

As long as the waiting time of the oldest packet of the user is less than the deadline, the scheduler attempts to exploit the multiuser diversity and schedules the users in an energy efficient way. When the deadline is reached for the oldest packet in the buffer, the user is forced to transmit on the available channel. One limitation of the emptying buffer policy is that it becomes difficult to empty the buffer for the large buffer sizes due to limited duration of a time slot. However, for small buffer sizes, it is possible and simplifies the scheduling mechanism a lot.

We use a Markov decision process (MDP) to model and analyze the scheduler. We define some terms used in this work.

Definition 1 (Backlog State): The backlog state in a MDP is defined as the waiting time of the oldest unscheduled packet in the buffer.

We consider random arrivals in each time slot for all the users and model them as constant arrivals with random content size. It has been proven in large system limit that such a representation results in equivalent state space descriptions for both constant and random arrivals due to system level averaging of data scheduled in each state [8]. This model makes our state space description independent of the arrival process. The backlog state represents the number of random size packets waiting to be scheduled in the buffer. Arrivals are queued in a finite buffer of $n$ random size packets before transmission.

Definition 2 (Opportunistic Threshold): An opportunistic threshold $\kappa_{i}$ is defined as the minimum short-term fading value allowing for the state transition from state $i$ to state 1 .

It should be noted that computation of opportunistic thresholds for all the users depending on their respective backlogstates is usually not feasible in a multiuser environment because of the large state space. We consider an asymptotically large user system in this work. Therefore, the backlog-states of the users decouple and we can formulate the problem of energy efficient transmission in a multiuser system as an equivalent single user scheduling problem [9].

In the MDP description, the deadline constraint is reflected by the maximum number of states $n$. Forward state transition from a state $i$ to the next higher state $j$ occurs if no data is transmitted. As we model random arrivals in each time slot by a constant arrival of random size and assume identical deadline for all the arrived packets, $j$ always equals to $i+1$ when a user is not scheduled. Due to emptying buffer property, the backward state transition occurs always from state $i$ to state 1 and the scheduler schedules $i$ packets for transmission. In state $n$, the user can empty the buffer if fading is better than the threshold $\kappa_{n}$ like other states. Moreover, the user is allowed to transmit the packet reaching the deadline alone if fading is smaller than $\kappa_{n}$ and returns back to state $n$. Note that this event requires no explicit threshold computation as opportunistic threshold for this transmission equals zero.

In the MDP if a user is in state $i$, then the next state $j$ is determined according to transition probabilities $\alpha_{i j}$. Let $S_{t}$ be the state of the process at time $t$. Then, the state $S_{t+1}$ depends only on state $i$ and action $a(t)$ as a function of short-term fading at time $t$. The fading randomizes the state transitions. The resulting transition probabilities are given by

$$
\begin{aligned}
\alpha_{i j} & =\operatorname{Pr}\left\{S_{t+1}=j \mid S_{t}=i, a_{t}=a\right\} \\
& = \begin{cases}\operatorname{Pr}\left(f>\kappa_{i}\right) & \forall i, j=1 \\
\operatorname{Pr}\left(f \leq \kappa_{i}\right) & \forall i \neq n, j=i+1 \\
\operatorname{Pr}\left(f \leq \kappa_{i}\right) & i=n, j=n \\
0 & \text { else }\end{cases}
\end{aligned}
$$

$\alpha_{i j}=0$ implies impossible state transition for the scheduler. The state transition diagram is shown in Fig. 1.

We assume that the users exhibit independent fading processes. Furthermore, the proposed scheduler is independent of the other users' fading. The law of large numbers drives the proportion of users in state $i$ at time $t$ to be identical to $\operatorname{Pr}\left(S_{t}=i\right)$ in the large user limit. 


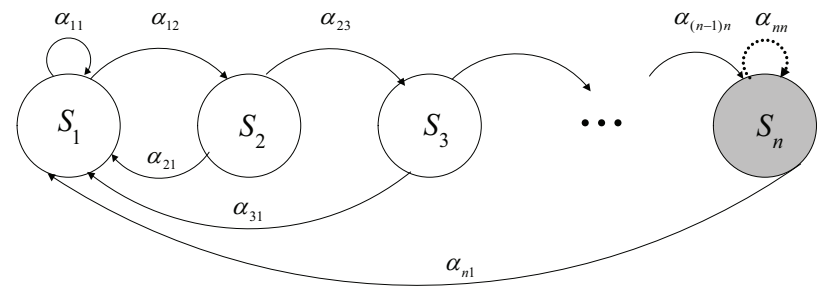

Fig. 1. State diagram for the transition states of the proposed scheduler.

\section{A. Large System Analysis}

To analyze the scheduler in large system limit, we apply the results of [7], [10]. For analysis purpose, we model a user that transmits multiple packets at a time as multiple virtual users with identical fading that transmit single packet. We denote the scheduled packets by virtual users (VU) here. For a single cell case, the average system energy per transmitted information bit at the large system limit $K \rightarrow \infty$ is then given by [10]

$$
\left(\frac{E_{\mathrm{b}}}{N_{0}}\right)_{\mathrm{sys}}^{\mathrm{SC}}=\log (2) \int_{0}^{\infty} \frac{2^{C \mathrm{P}_{g, \mathrm{VU}}(x)}}{x} \mathrm{dP}_{g, \mathrm{VU}}(x)
$$

where $\mathrm{P}_{g, \mathrm{VU}}(\cdot)$ denotes the cumulative distribution function (cdf) of the channel gains of the scheduled virtual users. It is composed of the short-term fading and the long-term fading of the VUs.

For the analysis of multicell case, we use the large system results from [7]. The average system energy per transmitted information bit at the large system limit $K \rightarrow \infty$ is given by

$$
\left(\frac{E_{\mathrm{b}}}{N_{0}}\right)_{\mathrm{sys}}^{\mathrm{MC}}=\frac{\left(\frac{E_{\mathrm{b}}}{N_{0}}\right)_{\mathrm{sys}}^{\mathrm{SC}}}{1-\beta C\left(\frac{E_{\mathrm{b}}}{N_{0}}\right)_{\mathrm{sys}}^{\mathrm{SC}}}
$$

where $\beta$ is a constant commonly used to model the effect of interference in multicell analysis and bounded by

$$
2 D^{-\alpha} \zeta(\alpha) \leq \beta \leq D^{-\alpha}\left(\zeta\left(\frac{1}{2} \alpha\right)+\zeta\left(\frac{3}{2} \alpha\right)\right)
$$

where $\zeta($.$) denotes the standard zeta function. It has been$ observed that $\beta$ does not change much with spectral efficiency and any $\beta$ within the bound can be used effectively to model the inter-cell interference [7].

As compared to single cell case, performance of multicell case behaves differently at large spectral efficiency. Performance degradation in multicell case is dependent on the behaviour of multiple cell interference. Depending on the multiple cell interference, we distinguish the following operating regions [7].

$$
\begin{cases}\left(\frac{E_{\mathrm{b}}}{N_{0}}\right)_{\mathrm{sys}}^{\mathrm{SC}} \leq \frac{1}{2 \beta C} & \text { noise dominated region } \\ \frac{1}{2 \beta C}<\left(\frac{E_{\mathrm{b}}}{N_{0}}\right)_{\mathrm{sys}}^{\mathrm{SC}}<\frac{1}{\beta C} & \text { interference dominated region } \\ \left(\frac{E_{\mathrm{b}}}{N_{0}}\right)_{\mathrm{sys}}^{\mathrm{SC}} \geq \frac{1}{\beta C} & \text { forbidden region }\end{cases}
$$

Performance degradation as compared to single cell case is up to $3 \mathrm{~dB}$ in the noise dominated region. When interference dominates the noise, performance degrading is more than $3 \mathrm{~dB}$. Contrast to single cell case, there is a certain limit on spectral efficiency $C$ beyond which it is not allowed to operate and this region is termed as forbidden region.

We evaluate probability distribution function (pdf) of the channel gain $\mathrm{p}_{g, \mathrm{VU}}(x)$ of the scheduled virtual users. The scheduling decisions affect the short-term fading distribution only. Following (6), the resulting pdf of the short-term fading of the VUs is given by

$$
\mathrm{p}_{f, \mathrm{VU}}(y)=\sum_{i=1}^{n} c_{i} \begin{cases}i \pi_{i} \mathrm{p}_{f}(y) & y>\kappa_{i}, \forall i \\ \pi_{n} \mathrm{p}_{f}(y) & y \leq \kappa_{i}, i=n \\ 0 & \text { else }\end{cases}
$$

where $\pi_{i}$ is the limiting probability of state $i$ while $\mathrm{p}_{f}(y)$ and $c_{i}$ denote the short-term fading and a constant to normalize the pdf. Equation (10) specifies that no data is scheduled for $y \leq \kappa_{i}$ and $i$ packets for $y>\kappa_{i}$ in all states other than $n$. The cdf of the VUs is given by

$$
\mathrm{P}_{f, \mathrm{VU}}(y)=\sum_{i=1}^{n} c_{i} \pi_{i} i\left(\mathrm{P}_{f}(y)-\mathrm{P}_{f}\left(\kappa_{i}\right)\right)+c_{n} \pi_{n} \mathrm{P}_{f}\left(\kappa_{n}\right) .
$$

$\mathrm{P}_{g, \mathrm{VU}}(y)$ is calculated using (11) and path loss distribution.

\section{B. Wideband Analysis at Low Spectral Efficiency}

We investigate the low spectral efficiency behaviour of the multicell systems specifically and derive an upper bound for the spectral efficiency at a given cell size. It has been shown that systems with the same $\left(E_{b} / N_{0}\right)_{\min }$ may have very different behaviours in the wideband regime and this behaviour can be expressed by evaluating wideband slope [11]. We denote the spectral efficiency as a function of $E_{b} / N_{0}$ by $C\left(E_{b} / N_{0}\right)$. Then, derivative of $C$ with respect to $E_{b} / N_{0}$ expressed in decibels, evaluated at $\left(E_{b} / N_{0}\right)_{\min }$ and normalized to $3 \mathrm{~dB}$ is called a wideband slope and denoted by $\mathcal{S}_{0}$ [10]. The low spectral efficiency behaviour is characterized by the minimum system $\left(E_{b} / N_{0}\right)_{\min }$ and the wideband slope $\mathcal{S}_{0}$ such that [10]

$$
\left.\left(E_{b} / N_{0}\right)_{\mathrm{sys}}\right|_{\mathrm{dB}}=\left.\left(E_{b} / N_{0}\right)_{\min }\right|_{\mathrm{dB}}+\frac{C}{\mathcal{S}_{0}} 10 \log _{10}(2)+\mathcal{O}(C) .
$$

Wideband slope for the multicell system is computed in Appendix A and given by

$$
\mathcal{S}_{0}=\frac{\int_{0}^{\infty} \frac{\mathrm{dP}_{\mathrm{g}, \mathrm{VU}}(\mathrm{x})}{x}}{\int_{0}^{\infty} \frac{P_{g, \mathrm{VU}}(x)}{x} \mathrm{dP}_{\mathrm{g}, \mathrm{VU}}(\mathrm{x})+\beta\left(\int_{0}^{\infty} \frac{\mathrm{dP}, \mathrm{VU}(\mathrm{x})}{\mathrm{x}}\right)^{2}}
$$

In the following, we determine an upper bound on spectral efficiency in terms of cell size by using the lower bound for $\beta$ and (13) in (12). For small $C$, (12) can be written as

$$
\left.\left(E_{b} / N_{0}\right)_{\mathrm{sys}}\right|_{\mathrm{dB}} \geq\left.\left(E_{b} / N_{0}\right)_{\min }\right|_{\mathrm{dB}}+\frac{C}{\mathcal{S}_{0}} 10 \log _{10}(2) .
$$

Equation (14) holds because (8) is convex in $C$ due to the fact that (7) is convex, positive and monotonously increasing in $C$. 
The upper bound on capacity is achieved by using lower bound for $\beta$ in (14) and given by

$$
\begin{aligned}
C \leq & \left(\left.\left(E_{b} / N_{0}\right)_{\mathrm{sys}}\right|_{\mathrm{dB}}-\left.\left(E_{b} / N_{0}\right)_{\min }\right|_{\mathrm{dB}}\right) \\
& {\left[1 0 \operatorname { l o g } _ { 1 0 } ( 2 ) \left(2 \zeta(\alpha) D^{-\alpha} \int_{0}^{\infty} \frac{\mathrm{dP}_{\mathrm{g}, \mathrm{VU}}(\mathrm{x})}{x}\right.\right.} \\
+ & \left.\left.\frac{\int_{0}^{\infty} \frac{P_{g, \mathrm{VU}}(x)}{x} \mathrm{dP}_{\mathrm{g}, \mathrm{VU}}(\mathrm{x})}{\left(\int_{0}^{\infty} \frac{\mathrm{dP}_{\mathrm{g}, \mathrm{VU}}(\mathrm{x})}{x}\right)}\right)\right]^{-1}
\end{aligned}
$$

The upper bound specifies the maximum spectral efficiency achievable at a given cell size $D$ in the presence of multicell interference. We compare the results from the bound and the maximum achievable capacity using (8) in Section V.

\section{OPTIMIZATION OF THRESHOLDS}

The proposed scheduler requires offline computation of optimal threshold for every state $i$. However, the system energy is not a convex function of the opportunistic thresholds. Therefore, we require some heuristic optimization technique to optimize the opportunistic thresholds. To evaluate the performance of the scheduler, we use the Simulated Annealing (SA) algorithm to optimize the energy function for the thresholds. Other similar algorithms like random search, genetic algorithm, etc., can also be used. The choice of SA is based solely on its wide acceptance in literature. It is believed to provide near optimal solutions for non convex stochastic optimization problems like traveling salesman problem. We skip the details of the algorithm due to space limitation and the interested reader is referred to [8], [12] for details.

\section{Numerical Results}

We consider a multi-access channel with $M$ bands having statistically independent fading on these channels. Every user senses $M$ channels and selects her best channel as a candidate for transmission. The users are placed uniformly at random in a cell except for a forbidden region around the access point of radius $\delta=0.01$. The path loss exponent $\alpha$ equals 2 and path loss distribution follows the model described in [7]. We assume exponential fast fading distribution with mean one for every user on each of the $M$ channels.

Table I shows the optimized thresholds for a single cell scheduler for different deadlines. As energy function for multicell case is a scaled version of energy function of a single cell case, the set of optimized transmission thresholds remains the same for both of the cases.

We quantify the energy-delay tradeoff exhibited by the scheduler for the single and multicell cases in Fig. 2 using (7) and (8). As expected, energy efficiency of the system improves as the packet deadline increases. We evaluate the effect of intercell interference on the system performance at different spectral efficiency values. As compared to single cell case, loss in multicell system energy is small at small spectral efficiencies. As the spectral efficiency increases, loss increases.
TABLE I

Threshold COMPUTATION FOR $M=1$ AND $C=0.5 \mathrm{bits} / \mathrm{s} / \mathrm{Hz}$

\begin{tabular}{|c||c||c||c||c|c|}
\hline$n$ & $\kappa_{1}$ & $\kappa_{2}$ & $\kappa_{3}$ & $\kappa_{4}$ & $E_{b} / N_{o}$ \\
\hline 2 & 0.24 & 0.24 & NA & NA & $-3.17 \mathrm{~dB}$ \\
3 & 0.50 & 0.32 & 0.32 & NA & $-4.72 \mathrm{~dB}$ \\
4 & 0.68 & 0.56 & 0.38 & 0.39 & $-5.68 \mathrm{~dB}$ \\
\hline
\end{tabular}

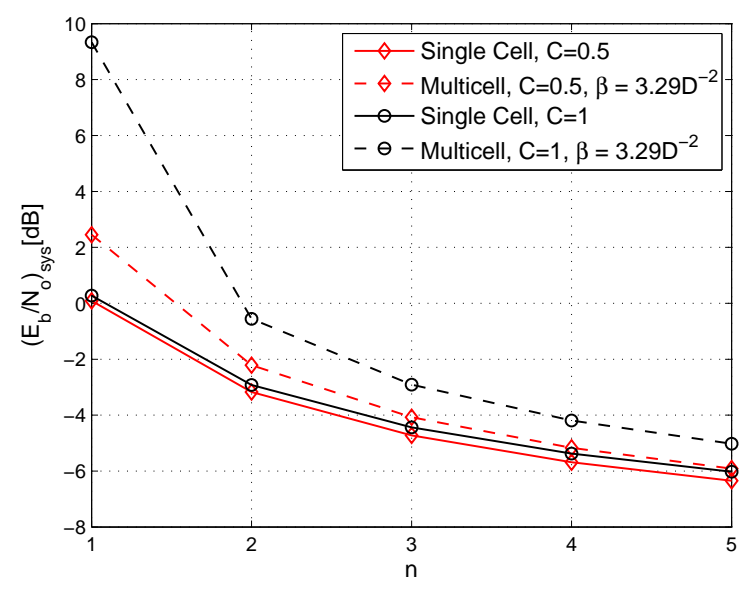

Fig. 2. Energy-delay tradeoff for the single and multicell cases with $M=1$ and $D=2$.

We demonstrated in Fig. 2 that delay tolerance makes the system energy efficient which has an other inherent advantage for the multicell case. It allows the system to operate at high spectral efficiency. In Fig. 3, we demonstrate the significance of delay tolerance for the multicell case. For the single cell case, there is no limit on the operating range of the spectral efficiency. However, as explained in Section III-A, the system cannot operate in forbidden region for the multicell case. The delay tolerance of the system widens the range of spectral efficiency values where the system can operate. In Fig. 3, spectral efficiency enters the forbidden region at smaller value for $n=1$ as compared to the system with $n=2$.

In Fig. 4, we study the behaviour of the capacity bound for the single and multicell cases derived in Section III-B and [10], respectively as a function of $D$ and fixed system energy. The capacity decreases as a function of $D$ for the both single and multicell cases. We observe the convergence of multicell capacity to single cell capacity due to negligible effect of the intercell interference on the system capacity at large cell sizes. For the same fixed system energy, we plot the capacity for the single and multicell cases using (7) and (8) as well. The minimum system energy value (used in evaluation of (15)) is $D$ specific and varies greatly from $-11 \mathrm{~dB}$ for $D=2$ to 2.78 $\mathrm{dB}$ for $D=10$. We choose the fixed system energy equals to $3 \mathrm{~dB}$ for all $D$ in the numerical example. There appears a significant gap between the capacity bounds and the computed capacity at small $D$ but this gap is due to large values of $C$ obtained in the bound at the fixed system energy of $3 \mathrm{~dB}$. The capacity bounds at large $C$ are loose due to ignoring the term $\mathcal{O}(C)$ in (14). At large $D$, the bound results in a small $C$ when the difference between the fixed system energy and the minimum system energy is small and the effect of ignoring $\mathcal{O}(C)$ is minimal. As illustrated in Fig. 3 as well, the computed 


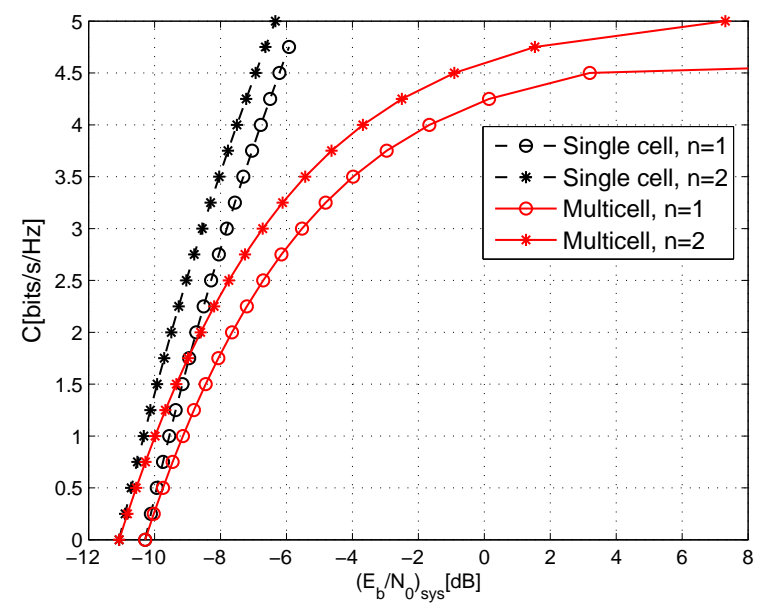

Fig. 3. Characterization of operating region for the proposed scheduler in a multicell scenario for the delay limited $(n=1)$ and the deadline constrained ( $n=2$ ) systems by using lower bound of $\beta, M=10$ and $D=2$.

capacity saturates at $5 \mathrm{bits} / \mathrm{s} / \mathrm{Hz}$ for $D=2$ regardless of the large system energy. Thus, the large gap between the computed capacity and the bound in this region is mere the result of ignoring the term $\mathcal{O}(C)$ in the computation of the bound.

\section{CONCLUSION}

We propose an opportunistic scheduling scheme which guarantees packet transmission before the deadline at low energy. The main contribution of the work is the large system analysis of the proposed scheme for the hard deadline constrained multiuser multicell systems. We evaluate the effect of the intercell interference on the system energy and determine an upper bound on the spectral efficiency for a given cell size using wideband analysis. We characterize the operating region for the delay constrained multicell system. The numerical results show how delay tolerance inherently helps to minimize the effect of intercell interference and increases the system operating region as compared to the delay limited systems.

\section{APPENDIX}

We compute wideband slope for the multicell system by

$$
\mathcal{S}_{0}=\log 2 \frac{f(0)}{f^{\prime}(0)} \text {. }
$$

where $f(0)$ denotes $\left(\frac{E_{\mathrm{b}}}{N_{0}}\right)$ in $(8)$ as a function of spectral efficiency $C$ and evaluated at $C \rightarrow 0$.

Evaluating (8) at $C \rightarrow 0$ gives

$$
f(0)=\log (2) \int_{0}^{\infty} \frac{\mathrm{dP}_{\mathrm{g}, \mathrm{VU}}(\mathrm{x})}{x}
$$

Differentiating (8) and evaluating at $C \rightarrow 0$ yields

$$
\begin{array}{r}
f^{\prime}(0)=\log (2)\left[\int_{0}^{\infty} \frac{\mathrm{P}_{\mathrm{g}, \mathrm{VU}}(\mathrm{x})}{x} \mathrm{dP}_{\mathrm{g}, \mathrm{VU}}(\mathrm{x})\right. \\
\left.+\beta\left(\int_{0}^{\infty} \frac{\mathrm{dP}_{\mathrm{g}, \mathrm{VU}}(\mathrm{x})}{x}\right)^{2}\right]
\end{array}
$$

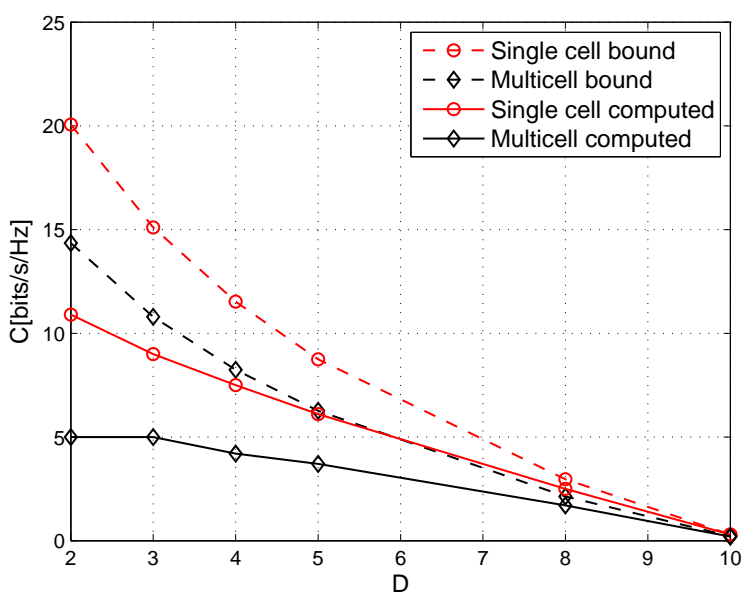

Fig. 4. Wideband approximation of capacity as a function of cell size for different values of $D, n=2, M=10$ and fixed $\left(E_{b} / N_{0}\right)_{\text {sys }}=3 \mathrm{~dB}$.

Using (17) and (18) in (16) gives wideband slope in (13).

\section{ACKNOWLEDGMENT}

The author would like to thank Kimmo Kansanen for the useful discussions regarding this work.

This work was carried out during an ERCIM "Alain Bensoussan" Fellowship tenure. This Programme is supported by the Marie Curie Co-funding of Regional, National and International Programmes (COFUND) of the European Commission.

\section{REFERENCES}

[1] D. Wu and R. Negi, "Utilizing multiuser diversity for efficient support of quality of service over a fading channel," IEEE Tansactions on Vehicular Technology, vol. 54, no. 3, pp. 1198-1206, May 2005.

[2] N. Chen and S. Jordan, "Downlink scheduling with guarantees on the probability of short-term throughput," IEEE Trans. Wireless. Comm., vol. 8, no. 2, pp. 593-598, 2009.

[3] A. Tarello, J. Sun, M. Zafar, and E. Modiano, "Minimum energy transmission scheduling subject to deadline constraints," Wireless Networks, vol. 14, no. 5, pp. 633-645, October 2008.

[4] J. Lee and N. Jindal, "Energy-efficient scheduling of delay constrained traffic over fading channels," IEEE Trans. Wireless Communications, vol. 8, no. 4, pp. 1866-1875, April 2009.

[5] T. P. Coleman and M. Medard, "A distributed scheme for achieving energy-delay tradeoffs with multiple service classes over a dynamically varying channel," IEEE journal on selected areas in communications, vol. 22, no. 5, pp. 929-941, june 2004.

[6] M. M. Butt, K. Kansanen, and R. R. Müller, "Provision of maximum delay guarantee at low energy in a multiuser system," in 10th International Symposium on Spread Spectrum Techniques and Applications (ISSSTA), Bologna, Italy, August 2008.

[7] D. Park and G. Caire, "Hard fairness versus proportional fairness in wireless communications: The multiple-cell case," in IEEE International Symposium on Information Theory, Toronto, Canada, jul. 2008.

[8] M. M. Butt, K. Kansanen, and R. R. Müller, "Hard deadline constrained multiuser scheduling for random arrivals," in WCNC, Cancun, Mexico, March 2011.

[9] P. Viswanath, D. N. Tse, and V. Anantharam, "Asymptotically optimal water-filling in vector multiple-access channels," IEEE Trans. Inform. Theory, vol. 47, no. 1, pp. 241-267, January 2001.

[10] G. Caire, R. Müller, and R. Knopp, "Hard fairness versus proportional fairness in wireless communications: The single-cell case," IEEE Trans. Inform. Theory, vol. 53, no. 4, pp. 1366-1385, April 2007.

[11] S. Verdu, "Spectral efficiency in the wideband regime," IEEE Trans. Inform. Theory, vol. 48, no. 6, pp. 1319 -1343, june 2002.

[12] S. Kirkpatrick, C. Gelatt, and M. Vecchi, "Optimization by simulated annealing,” Science, vol. 220, no. 4598, pp. 671-680, May 1983. 